Article

\title{
Fluoropolymer-Wrapped Conductive Threads for Textile Touch Sensors Operating via the Triboelectric Effect
}

\author{
Morgan Baima and Trisha L. Andrew * (1D \\ Departments of Chemistry and Chemical Engineering, University of Massachusetts Amherst, \\ Amherst, MA 01003, USA; morgan.baima@gmail.com \\ * Correspondence: tandrew@umass.edu; Tel.: +1-413-545-1651
}

Received: 3 May 2018; Accepted: 6 June 2018; Published: 11 June 2018

\begin{abstract}
Touch-sensitive electrical arrays are the primary user interface for modern consumer electronics. Most contemporary touch sensors, including known iterations of textile-based touch sensors, function by detecting capacitive changes within a circuit resulting from direct skin contact. However, this method of operation fails when the user's skin or the surface of the touch sensor is dirty, oily or wet, preventing practical use of textile-based touch sensors in real-world scenarios. Here, an electrically touch-responsive woven textile is described, which is composed of fluoropolymer-wrapped conductive threads. The fluoropolymer wrapping prevents contaminant buildup on the textile surface and also electrically insulates the conductive thread core. The woven textile touch sensor operates via surface potential changes created upon skin contact. This method of operation, called the triboelectric effect, has not been widely used to create textile touch sensors, to date. The influences of surface wetness and varying skin surface chemistry are studied, and the triboelectric textile touch sensors are found to be advantageously insensitive to these environmental variables, indicating that triboelectric textiles have promise for practical use as touch interfaces in furniture and interior design.
\end{abstract}

Keywords: textile electronics; plain weave; touch sensor; triboelectricity

\section{Introduction}

Touch-sensitive electronic devices serve as the primary user interface for a plethora of current technologies, such as portable computers, e-readers, smart phones, smart watches, fitness trackers, in-vehicle consoles, interactive display screens and touchpads [1]. Two main types of touch sensitive screens are used in these technologies: resistive touch sensors and capacitive touch sensors $[1,2]$. Resistive touch sensors are composed of two conductive sheets, arranged face-to-face and physically separated by a micron-length air gap. When a user touches this type of device, the two conductive sheets are physically brought into contact with each other due to the force associated with the touch interaction, and an electrical signal is recorded and processed by a relevant operating system. Capacitive touch screens are comprised of a single insulating substrate, such as glass or poly(ethylene terephthalate), coated with a patterned array of conductive electrodes on one side. A small voltage is applied across this electrode array, creating a uniform electric field across the opposite, uncoated surface of the insulating substrate. When a user touches this uncoated side, a capacitor is dynamically formed across the electrode-insulator-finger arrangement, since human skin is conductive. The dynamic change in device capacitance is then registered by a controller.

While selected flexible, touch-sensitive device architectures are known [3,4], translating these devices to fiber-based substrates is not straightforward. Selected touch-responsive textiles that produce 
a resistance change when an exposed conductive thread comes into direct contact with human skin have been previously reported [5-12]. However, this method of operation fails when the user's skin or the surface of the touch sensor is dirty, oily or wet, preventing practical use of textile-based touch sensors in real-world scenarios.

Recently there has been increased scientific interest in triboelectric generators (TEGs) due to their ability to convert small force inputs into an electrical (voltage and current) output. Because these devices operate by detecting the surface potential changes created upon contact and release of dissimilar surfaces (due to either the triboelectric effect or contact electrification), simple adjustments of device architecture can provide a wide range of functional technologies [13-15]. Here, we use the triboelectric effect (or, similarly, the contact electrification effect) to transduce touch events into electrical signals using a woven textile composed of fluoropolymer-wrapped [16] conductive fibers. The fluoropolymer wrapping prevents contaminant build up on the textile surface and also electrically insulates the conductive thread core. Triboelectric textile touch sensors are found to be advantageously insensitive to common environmental variables, indicating their promising use as touch interfaces in furniture and interior design.

\section{Materials and Methods}

Device Assembly: Threads used in this study were either stainless steel 2-ply conductive thread purchased from Adafruit (product 640) (New York City, NY, USA) with a reported resistivity of $51 \mathrm{Ohm} / \mathrm{m}$ and diameter of 200 micrometers, or 2-ply conductive silver-plated nylon thread purchased from LessEMF (Cat.\#A304) (Latham, NY, USA) with a reported resistivity of $2 \mathrm{kOhm} / \mathrm{m}$ and a diameter of approximately 140 micrometers. The poly(tetrafluoroethylene) (PTFE) tape used in this study was low density ( 0.35 to 0.5 specific gravity) thread sealant tape with a width of 0.5 inches and a thickness of 88.9 nanometers. All woven devices were fabricated by folding the PTFE tape in half over the conductive thread or threads, and then twisting the tape with an electric drill. For clarity, these tape-covered threads are referred to as "strings". The total weight of each of the PTFE-wrapped strings was less than $5 \%$ greater than the starting mass of the naked conductive thread. Textile touch sensors were hand-woven on a small loom (Beadsmith brand) using a plain weave pattern, using one string as the warp and one string as the weft, or $20 \times 60$ strings in total.

Electrical Characterization: All measurements were performed using a Wavenow potentiostat/ galvanostat by Pine Research or a Tektronix three-channel oscilloscope. Voltage outputs were obtained using the open circuit potential experiment and current outputs were obtained using the chronoamperometry experiment with the applied voltage set to zero volts. Conductive threads from either the warp or the weft were attached to the potentiostat using an alligator clip. "Dry" tests were performed by placing the devices in a sealed dessicator with Drierite dessicant overnight. Nitrogen gas was blown on the samples before storage in the dessicator and during the data collection upon immediate removal from the dessicator. Wet tests (deionized water, tap water, and $\mathrm{NaCl}$ salt solutions) were performed by spraying the devices with the respective wet solvent or solution and testing while the surface of the device was wet. For the $\mathrm{NaCl}$ salt solutions, $40 \mathrm{mmol} / \mathrm{L}$ solutions were made using deionized (D.I.) and tap water, respectively, as the solvent in order to mimic human sweat. For "wet hand" tests, the individual executing the test sprayed his or her hand with the wet solvent or solution, and then collected the data from the wet skin test onto a dry device. Samples were rinsed with deionized (D.I.) water and patted dry between all wet tests. Samples were also air-dried for five minutes after patting dry. Lotion tests were performed by applying lotion to both the user's hand and the sensor surface (separately). For the lotion-on-hand tests, a generous amount ( $0.32 \mathrm{~g}$, allowed to absorb, and then re-applied for a total of $0.64 \mathrm{~g}$ ) of Lubriderm Daily Moisture for normal to dry skin was applied to the hands and rubbed into the skin. To prevent extensive absorption, no wait time was allowed, and tests were carried out immediately after applying the lotion. For the lotion-on-sensor tests, a $0.32 \mathrm{~g}$ aliquot of lotion was spread on the sensor surface and repeated two times. The lotion 
was then wiped away from the sensor surface using a paper towel doused in either isopropanol or Clorox Green Works All-Purpose Cleaner.

\section{Results and Discussion}

\subsection{Fluoropolymer-Wrapped Conductive Threads}

Human skin is, on average, considered to possess a positive surface charge [1]. Therefore, to dynamically create a triboelectric generator upon skin contact, a complementary dielectric material carrying negative surface charge is needed. Poly(tetrafluoroethylene) (PTFE), a fluoropolymer, was chosen as the negative triboelectric material due to the strong electron affinity demonstrated by fluorinated polymers [17]. Furthermore, PTFE is a longstanding protective coating that prevents the acid/salt corrosion of, and biofilm formation on, various electronic components. In order to create a textile touch sensor, conductive threads were, first, tightly wrapped with PTFE tape (which is widely available because of its use as a waterproof, grease-free joint sealant in household plumbing) using an improvised yarning technique. Any commercially-available conductive thread can be used; in this report, two-ply stainless-steel yarns or silver-coated nylon yarns were used as the conductive core. Upwards of 40 feet of PTFE-covered thread are produced per yarning run. All exposed surfaces of the conductive thread were completely covered with PTFE tape, and no conductive surfaces were exposed along the length of the thread, with the exception of the ends that were left bare to allow electrical contact. The PTFE tape was in tight physical contact with the conductive thread core, allowing surface charge collection and transport to an external circuit. These PTFE-covered conductive threads were then plain woven using a tabletop loom to create a textile touch sensor.

\subsection{Plain-Woven Triboelectric Textile Touch Sensors}

Figure 1 illustrates the straightforward assembly of a plain-woven textile touch sensor created using PTFE-wrapped conductive threads. A picture of the completed 5 inch $\times 5$ inch textile is provided in Figure 1b, and the operative mechanism for detecting skin contact is illustrated in Figure 1c. When the positively-charged surface of skin comes into contact with the negatively-charged PTFE surface of the textile, the equilibrium surface potential of the textile surface is perturbed due to surface charge transfer between the skin and the PTFE, and a compensating inductive current is generated through the conductive thread core. Upon separation of the skin from the PTFE, surface charge transfer ceases and a compensating current is created in the reverse direction to restore the equilibrium surface charge distribution of the textile.

The voltage generated by a touch action is determined by the amount of extra surface charges created upon skin/textile contact. Voltages between 4 and 5 volts are typically generated with a load resistance of $50 \mathrm{M} \Omega$ when a clean, dry finger contacts the dry textile touch sensor (or 1-2 V with a $500 \mathrm{G} \Omega$ load resistance). The current output is proportional to the amount of surface charge collected by the conductive thread core. A few microamperes of current are typically produced upon skin contact. The current output resulting from a single touch-release action on the woven textile touch sensor is depicted in Figure $1 \mathrm{~d}$.

Each warp thread in the woven textile touch sensor can, in theory, be connected to an independent sensing channel of a controller, which will only register an electrical signal when a user touches the part of the textile containing this thread. For this study, an oscilloscope with three independent channels was used and, therefore, the bare ends of the warp threads of the textile were portioned and bundled together to form three spatially-distinct (lengthwise) channels, labelled Region 1, 2, and 3 (Figure 2). A movie showing the electrical output from touching actions is provided in the Supporting Information (Video S1). Figure 2b shows groups of pulses generated in three different oscilloscope channels upon repeated touch-release actions performed on different spatial regions of the textile sensor. Each of the three channels operates independently and only manifests an electrical output when the corresponding region of the textile is touched. Therefore, this textile touch sensor automatically demonstrates spatial 
resolution concomitant with the weave density (or fill) used to create the device. A looser weave density would result in lower spatial resolution of touch events; therefore, only tight-woven textiles were investigated here. Further, when a finger is swiped across a large region of the textile, the shape of the electrical output is notably different and multiple channels record the dragging motion.

(a)

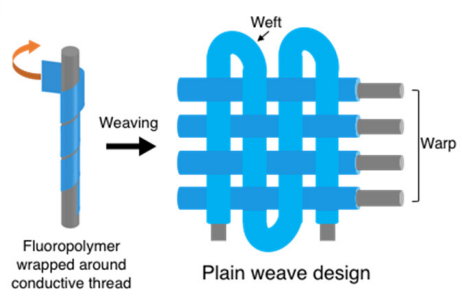

(b)

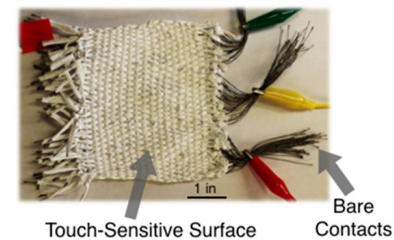

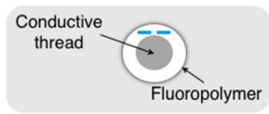
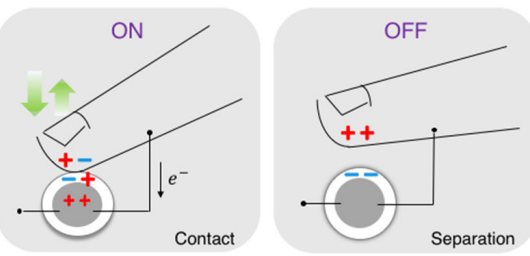

(d)

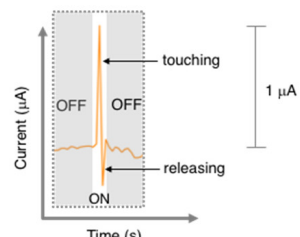

Figure 1. (a) Illustrated assembly of a woven textile touch sensor. The fluoropolymer is colored differently in the illustration for clarity. (b) Optical image of completed device. (c) Surface potential changes caused by skin contact and release. Not depicted to scale. (d) Current output of the textile touch sensor for a single touch and release action. The time duration of the "ON" signal is directly related to the duration of skin contact on the textile.

(a)
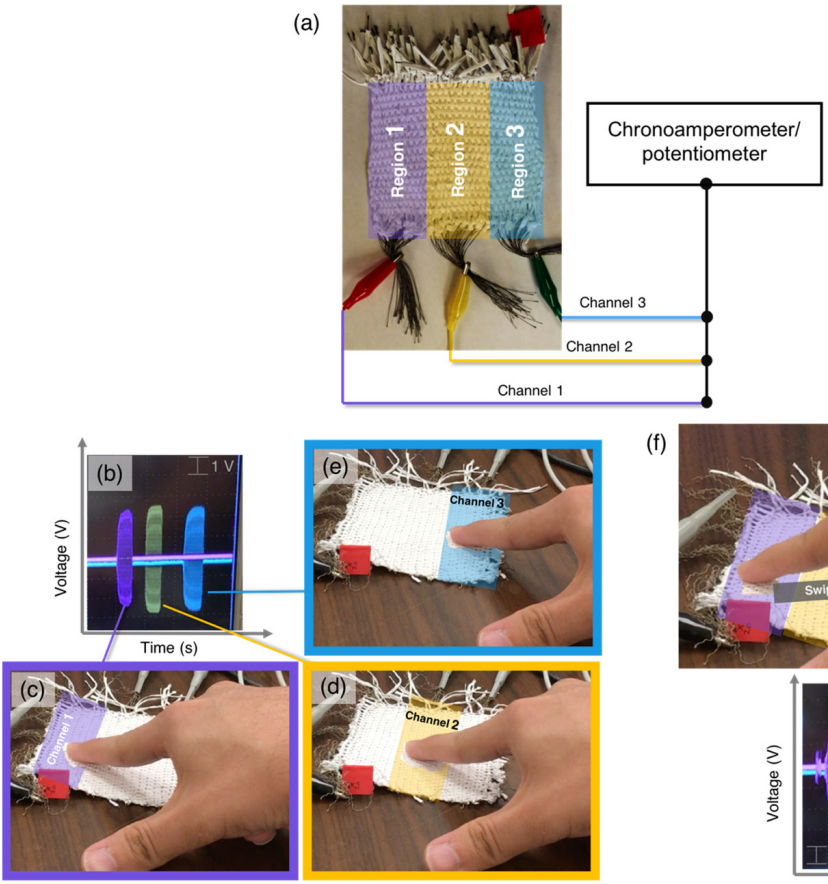

(f)
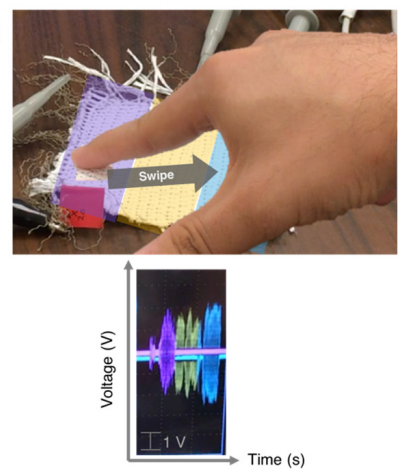

Figure 2. (a) Circuit diagram for testing woven textile touch sensors. The conductive threads from each string are bundled and connected to the potentiometer/chronoamperometer, segmenting the device into separate readout channels indicated by the different colored areas. (b) Voltage output with a $500 \mathrm{G} \Omega$ load resistance from three different sensing channels (purple, yellow, blue) when the corresponding spatial regions of the textile sensor (highlighted) are touched (c-e). (f) Voltage output with a $500 \mathrm{G} \Omega$ load resistance when a finger is swiped across the length of the textile touch sensor. 
Current output is, generally, the limiting metric for all triboelectric devices, which otherwise generate remarkably high open-circuit voltages [13-16]. Therefore, in this study, the current output of the textile was primarily used as the distinguishing metric with which to identify touch events under various conditions.

Gestural differences result in varying current output from the textile touch sensor (Figure 3). Different touch interactions exert different characteristic forces on a surface [3,4]. A greater applied force results in a larger surface area of skin-textile contact, creating greater triboelectrification/contact electrification and resulting in an increased peak magnitude under short circuit conditions. The average current output created by a variety of common touch gestures on the woven textile sensor are plotted in Figure 3. Events with smaller applied force, such as swiping the hand across the device or touching softly, resulted in smaller short-circuit current outputs than harder slaps or pounds. Nevertheless, lighter touching actions still produced sizeable current output (few microamperes) that would be sufficiently detectable by existing electronic circuitry for interactive sensing applications.

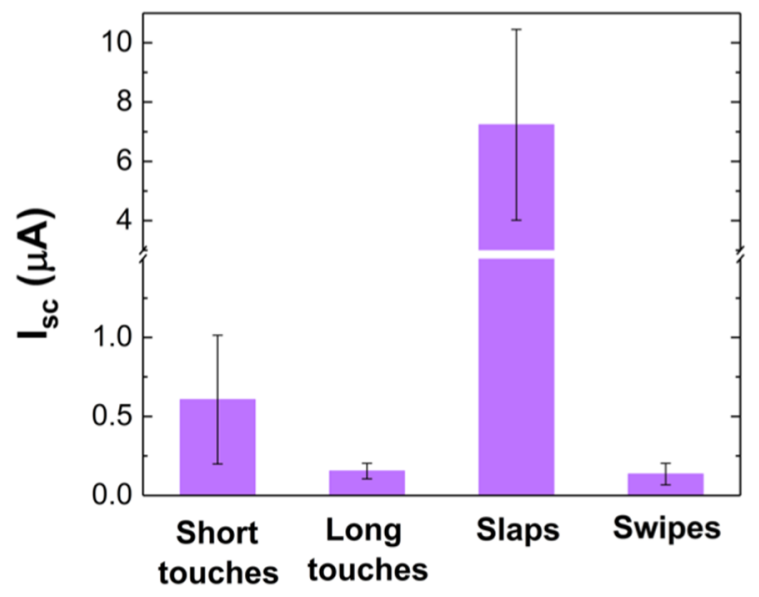

Figure 3. Current output under short circuit conditions for different gestures. Short touches and slaps lasted approximately $0.5 \mathrm{~s}$, while long touches lasted approximately 1 to $1.5 \mathrm{~s}$, and swipes lasted approximately $2 \mathrm{~s}$.

Touch sensors are typically operated under dirty, "real-world" conditions where grease, sweat, salt, water and/or other biofilms accumulate on the surface of the sensor. Therefore, it is necessary to understand how the electrical output of textile touch sensors is affected by common environmental variables, such as water and sweat, that can be present on the surface of the interactor's skin. In order to assess the performance of the textile touch sensors in humid and sweaty environments, the current output under short circuit conditions was tested in two different ways: by wetting the sample surface and testing with dry hands and by using wet/salty hands to touch a dry sample surface. Both of these testing methods were explored because the PTFE wrapping effectively protects the conductive thread cores of the textile and prevents salt/biofilm accumulation on the textile surface.

Minimal changes in the average current output from the textile touch sensor were observed for either wet, dry or oily testing conditions (Figure 4). The observed voltage outputs from the textile touch sensor were similarly insensitive to the particular wetness or dirtiness of the surface or of the user's hand (Figure S1), although a globally-lowered voltage output was observed in the presence of excess water or ions, likely because skin contact-created surface charges were rapidly dissipated into the aqueous or ionic environment [17]. Similarly, wet surfaces and wet/salty hands resulted in a greater standard deviation in the magnitude of the observed current output (Figure $4 b, c)$ but the average current output value was similar to the average current output when a pristine, dry textile was touched with clean, dry hands. 
The two exceptions were the noticeably lower current output generated when the textile touch sensor was touched with a finger dipped into a salt water solution made with tap water or with lotion-saturated skin. Only the current output was affected in both cases, and the voltage outputs remained unchanged compared to other testing conditions. Further, fingers dipped in brine made with distilled water did not yield a lowered current output. We posit that these tap water and lotion outliers likely arise due to mechanical disruptions-the salty tap water and lotion make the surface of the user's skin especially sticky and subsequent touch interactions jostle the electrical connections attached to the textile touch sensor. This likely causes an increase in thread resistance that manifests as a lowered current output.

Overall, surface wetness and wet/sweaty fingers did not meaningfully change the current output generated by the textile touch sensor, qualifying this device for real-world operability.
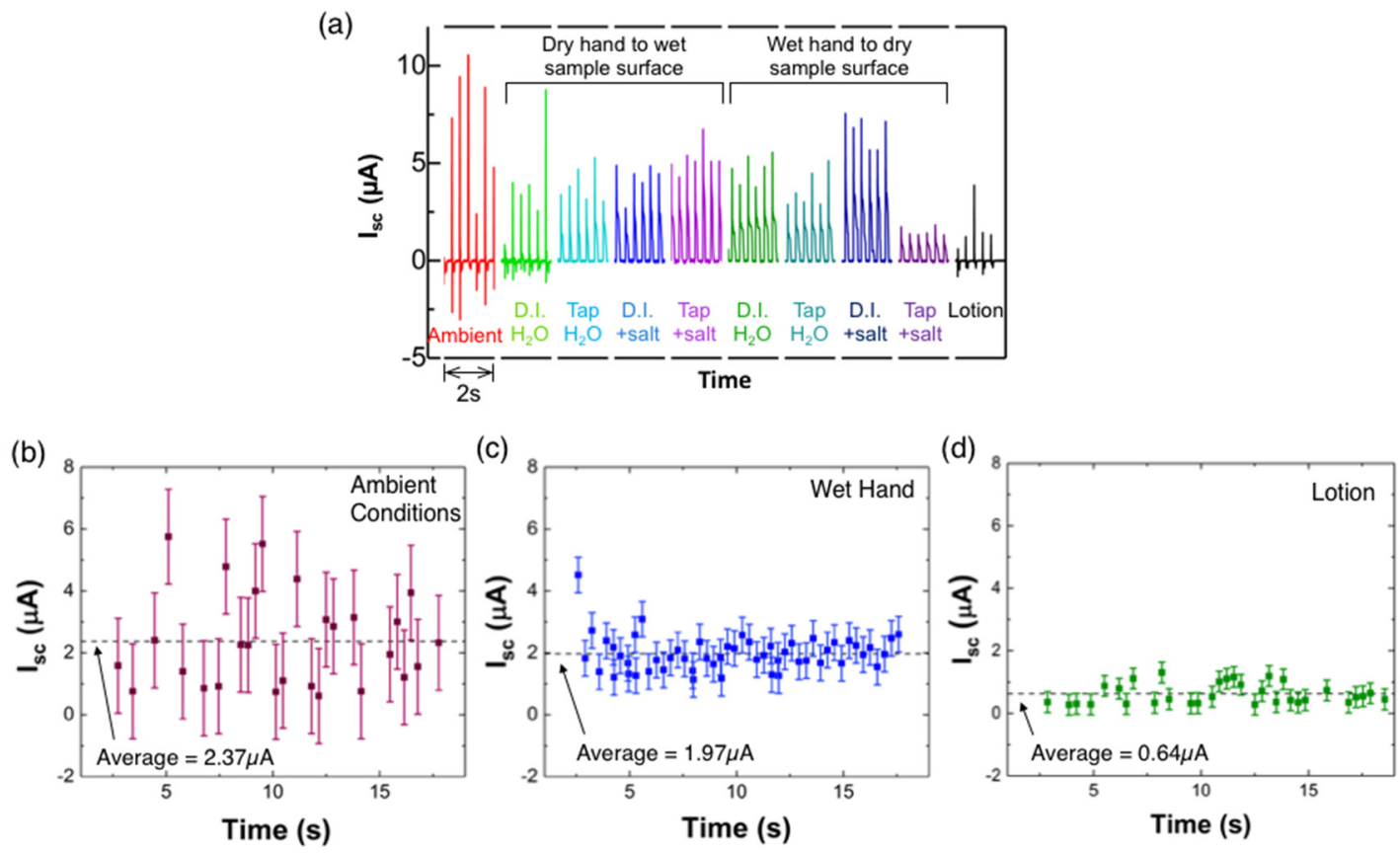

Figure 4. (a) Current output generated upon repeated skin contact and separation under different testing conditions. Peak values of current output with standard deviations for (b) Normal (dry sample, dry hands), (c) Wet hand touch actions, and (d) Touches with a small amount of lotion on skin. The dashed lines indicate the average peak value for each case.

To further explore the extent to which surface-accumulated oils affect the current output of the touch-responsive fabrics, a controlled sequence of experiments was performed before, during, and after lotion had been applied to the sensor's surface (not the user's hand) and subsequently wiped away using either a commercial cleaning product or isopropanol. Current outputs resulting from gentle touch actions ( $0.5 \mathrm{~s}$ duration) were recorded after each of the following actions were taken (corresponding to the experiment numbers depicted in Figure 5): (1) hands were washed with soap and water and a normal, dry sensor surface was touched; (2) excess lotion was applied to the skin surface and the sensor surface was touched; (3) lotion was coated on the sensor surface and then touched; (4) a second aliquot of lotion was applied to the sensor surface and touched; (5) hands were washed with soap and water and the sensor was touched; (6) the sensor surface was wiped with a paper towel doused with isopropanol and then touched; (7) the sensor surface was wiped again with another paper towel doused with isopropanol and then touched; and (8) the sensor surface was wiped a third time with a new paper towel doused with a commercial cleaning product. 
The textile touch sensor showed an approximately ten-fold decrease in average current output when an excessive amount of lotion (representing extreme oil buildup) was present on the device surface but returned to normal functionality after the lotion was cleaned from the device surface using either a commercial cleaner or isopropanol. These results suggest that surface oils interfere slightly with the triboelectric effect, but not enough to prevent, or irreversibly alter, device operation. The maximum current output peak values and average current output peak values with standard deviations for these experiments are shown in Figure 5.

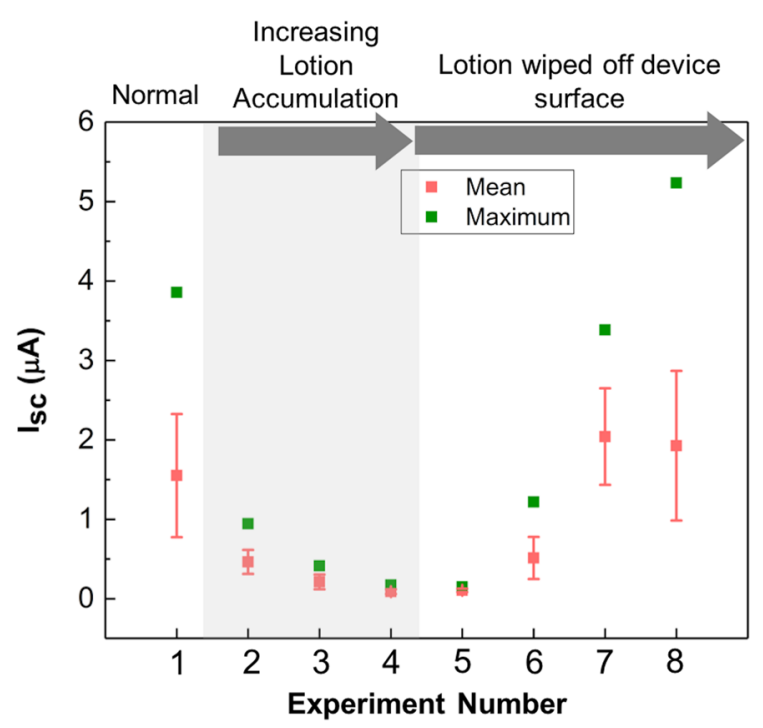

Figure 5. Maximum and mean values of current output generated upon repeated touch actions on a lotion-contaminated sensor surface. The experiment numbers are explained in the text.

\section{Conclusions}

The triboelectric effect was used to transduce touch events into an electrical signal using a plain-woven textile composed of fluoropolymer-wrapped conductive fibers. The textile touch sensor automatically demonstrated spatial resolution concomitant with the fill density. Gestural differences were also be identified by varying the current output from the textile touch sensor. The influences of surface wetness and varying skin surface composition were studied, and the textile touch sensors were found to be advantageously insensitive to these environmental variables. The woven textile touch sensors could foreseeably be implemented as a fabric touchpad to control smart garment functions or used as a control console in car seats and household furniture. Small patches of these touch-sensitive fabrics could also be sewn onto other backing textiles or incorporated into a garment, console, or furniture upholstery to serve as a fabric touchpad.

Supplementary Materials: The following are available online at http:/ / www.mdpi.com/2079-6439/6/2/41/s1, Figure S1: Voltage output of the woven textile touch sensor under different, dirty operating conditions, Video S1: WovenTextileTouchSensor_LowRes.mp4.

Author Contributions: M.B. and T.L.A. conceived and designed the experiments; M.B. performed the experiments; M.B. and T.L.A. analyzed the data; T.L.A. and M.B. wrote the paper.

Acknowledgments: Financial support by the David and Lucille Packard Foundation is gratefully acknowledged.

Conflicts of Interest: The authors declare no conflict of interest.

\section{References}

1. Walker, G. A review of technologies for sensing contact location on the surface of a display. J. Soc. Inf. Disp. 2012, 20, 413-440. [CrossRef]

2. Baxter, L.K. Capacitive Sensors: Design and Applications; Wiley: Hoboken, NJ, USA, 1996. 
3. Yuan, Z.; Zhou, T.; Yin, Y.; Cao, R.; Li, C.; Wang, Z.L. Transparent and flexible triboelectric sensing array for touch security applications. ACS Nano 2017, 11, 8364-8369. [CrossRef] [PubMed]

4. Pu, X.; Liu, M.; Chen, X.; Sun, J.; Du, C.; Zhang, Y.; Zhai, J.; Hu, W.; Wang, Z.L. Ultrastretchable, trasparent triboelectric nanogenerator as electronic skin for biomechanical energy harvesting and tactile sensing. Sci. Adv. 2017, 3, e1700015. [CrossRef] [PubMed]

5. Wijesiriwardana, R.; Mitcham, K.; Hurley, W.; Dias, T. Capacitive fiber-meshed transducers for touch and proximity-sensing applications. IEEE Sens. J. 2005, 5, 989-994. [CrossRef]

6. Vallett, R.; Young, R.; Knittel, C.; Kim, Y.; Dion, G. Development of a carbon fiber knitted capacitive touch sensor. MRS Adv. 2016, 1, 2641-2651. [CrossRef]

7. Roh, J.S. Textile touch sensors for wearable and ubiquitous interfaces. Text. Res. J. 2013, 84, 739-750. [CrossRef]

8. Ferri, J.; Lidon-Roger, J.V.; Morena, J.; Martinez, G.; Garcia-Breijo, E. A wearable textile 2D touchpad sensor based on screen-printing technology. Materials 2017, 10, 1450. [CrossRef] [PubMed]

9. Takamatsu, S.; Yamashita, T.; Imai, T.; Itoh, T. Fabric Touch Sensors using Projected Self-Capacitive Touch Technique. Sens. Mater. 2013, 25, 627-634.

10. Büscher, G.H.; Kõiva, R.; Schürmann, C.; Haschke, R.; Ritter, H.J. Flexible and Stretchable Fabric-Based Tactile Sensor. Robot. Autonom. Syst. 2015, 63, 244-252. [CrossRef]

11. Suen, M.; Lin, Y.; Chen, R. A Flexible Multifunctional Tactile Sensor using Interlocked ZnO Nanorod Arrays for Artificial Electronic Skin. Proc. Eng. 2016, 168, 1044-1047. [CrossRef]

12. Tu, H.; Chen, X.; Feng, X.; Xu, Y. A post-CMOS compatible smart yarn technology based on SOI wafers. Sens. Actuators A 2015, 233, 397-404. [CrossRef]

13. Wang, X.; Wang, Z.; Yang, Y. Hybridized nanogenerator for simultaneously scavenging mechanical and thermal energies by electromagnetic-triboelectric-thermoelectric effects. Nano Energy 2016, 26, 164-171. [CrossRef]

14. Lee, K.; Yoon, H.; Jiang, T.; Wen, X.; Seung, W.; Kim, S.; Wang, Z.L. Fully packages self-powered triboelectric pressure sensor using hemispere-array. Adv. Energy Mater. 2016, 6, 1502566. [CrossRef]

15. Wang, X.; Zhang, H.; Dong, L.; Han, X.; Du, W.; Zhai, J.; Pan, C.; Wang, Z.L. Self-powered high-resolution and pressure-sensitive triboelectric sensor matrix for real-time tactile mapping. Adv. Mater. 2016, 28, 2896-2903. [CrossRef] [PubMed]

16. Zhang, L.; Yu, Y.; Eyer, G.P.; Suo, G.; Kozik, L.A.; Fairbanks, M.; Wang, X.; Andrew, T.L. All-textile triboelectric generator compatible with traditional textile process. Adv. Mater. Technol. 2016, 1, 1600147. [CrossRef]

17. Thomas, S.W., III; Vella, S.J.; Dickey, M.D.; Kaufman, G.K.; Whitesides, G.M. Controlling the kinetics of contact electrification with patterned surfaces. J. Am. Chem. Soc. 2009, 131, 8746-8747. [CrossRef] [PubMed] 